\title{
Evaluación de la brecha de rendimiento para maíz tardío con distintas densidades de siembra en la región central de Córdoba, Argentina
}

\author{
de la Casa, A., Ovando, G., Bressanini, L., Díaz, G., Díaz, P. y Miranda, C.
}

\begin{abstract}
RESUMEN
A partir del modelo AquaCrop se evaluó la brecha de rendimiento (BR) en maíz de siembra tardía en la región central de Córdoba, Argentina, comparando densidades de siembra (DS) de 6 y $8 \mathrm{pl} \mathrm{m}^{-2}$. La ejecución del modelo entre 1960 y 2017 demandó analizar la consistencia del método de PenmanMonteith (PM) para estimar la tasa diaria de evapotranspiración de referencia (ETo) utilizando sólo registros de temperatura máxima y mínima (ETo_PM ${ }_{T \times T n}$ ), en lugar del set completo de cuatro variables fundamentales. Los valores de ETo_PM ${ }_{\text {TXTn }}$ explicaron $80 \%$ de la variabilidad de aquellos obtenidos con el conjunto completo de variables. AquaCrop se calibró a partir de muestras de cobertura del cultivo, biomasa aérea y agua del suelo obtenidas en un lote de maíz tardío durante el ciclo 2015-2016. Fueron evaluadas tres fechas de siembra fijas y tres variables, sin encontrar diferencias significativas de BR para ambas DS. El adelanto o atraso de la siembra no representó un manejo que altere la condición del riesgo climático. Aunque el incremento de DS a 8 pl $\mathrm{m}^{-2}$ aumentó significativamente la BR también alcanzó rendimientos efectivos (RR) medios más altos a los obtenidos con $6 \mathrm{pl} \mathrm{m}^{-2}$ en 47 de los 57 ciclos de cultivo analizados.
\end{abstract}

Palabras clave: AquaCrop; cobertura de cultivo; rendimiento potencial; riesgo.

de la Casa, A., Ovando, G., Bressanini, L., Díaz, G., Díaz, P. and Miranda, C., 2019. Yield gap assessment for late corn with different seeding densities in the central region of Córdoba, Argentina. Agriscientia 36 (2): 1-17

\section{SUMMARY}

The yield gap (BR) in late corn (December) was evaluated in the central region of Córdoba, Argentina, comparing planting densities (DS) of 6 and $8 \mathrm{pl} \mathrm{m}^{-2}$ using the AquaCrop model. Running the model between 1960 and 2017 required 
analyzing the consistency of the Penman-Monteith method (PM) to estimate the daily rate of reference evapotranspiration (ETo) using only maximum and minimum temperature records (ETo_PMTXTn), instead of the complete set of 4 fundamental variables. The ETo_PM ${ }_{\text {TxTn }}$ values explain approximately $80 \%$ of the variability of those obtained with the complete set of variables. AquaCrop was calibrated by using crop coverage samples, aerial biomass and soil water that were collected in a plot of late corn during the crop season 2015-2016. Three sowing dates were evaluated, 3 fixed and 3 variables, with no significant differences in BR for both DS. A small advance or delay in planting does not represent a decision affecting climate risk condition. While the increase of DS to $8 \mathrm{pl} \mathrm{m}^{-2}$ produced a significant increase in $\mathrm{BR}$, it also presented average effective yields (RR) that are higher than those obtained with $6 \mathrm{pl} \mathrm{m}^{-2}$ for 47 of the 57 crop cycles analyzed.

Keywords: AquaCrop; crop coverage; potential yield; risk.

de la Casa, A., Ovando, G., Díaz, G. y Díaz, P.: Agrometeorología de la Facultad de Ciencias Agropecuarias (FCA-UNC), Córdoba (CP 5000), Argentina. Bressanini, L.: Agrotecnia de la Facultad de Ciencias Agropecuarias (FCA-UNC). Miranda, C.: Establecimiento Las Delicias, Córdoba, Argentina. Correspondencia a: delacasa@agro.unc.edu.ar

\section{INTRODUCCIÓN}

El rendimiento potencial (RP) de un cultivo expresa la máxima productividad de un genotipo que crece y se desarrolla sin limitaciones de agua, nutrientes y otros factores reductores (insectos, malezas, etc.). La diferencia que se suscita cada año entre RP y el rendimiento real o efectivo (RR) constituye la brecha de rendimiento del cultivo (BR) (Lobell, Cassman y Field, 2009), cuya evaluación es importante a los efectos de diseñar las estrategias de manejo más conveniente en una región y conocer el nivel de riesgo involucrado en cada caso. Asimismo, su análisis permite diferenciar la eficiencia climática, cuando la brecha considera solo el efecto del déficit hídrico por lluvias insuficientes, de la eficiencia vinculada a un manejo sub-óptimo del cultivo (Battisti et al., 2018). En este sentido, la BR de un cultivo puede constituir un indicador apropiado a fin de expresar la aptitud de una región para una especie o variedad en particular, evaluar la eficacia de una práctica de manejo y calificar eventualmente el potencial productivo de cada año.

La reducción de BR del cultivo de maíz en secano es una meta difícil de alcanzar en el centro de la provincia de Córdoba, Argentina, cuyas condiciones sub-húmedas y la acentuada variabilidad interanual de la lluvia determinan que la falta de agua sea una contingencia presente con mayor o menor intensidad en la mayoría de los años. En respuesta a la habitual reducción de la productividad que la falta de agua ocasiona en secano, tanto por la insuficiencia y retraso de la lluvia como por tasas de evapotranspiración muy elevadas en verano, la demora de la siembra de maíz hasta el mes de diciembre se ha constituido los últimos años una modalidad generalizada en la región semiárida de la Argentina (Méndez, Casagrande y Vergara, 2014). Sin embargo, con el atraso de la fecha de siembra los niveles de radiación solar incidente y temperatura decaen progresivamente durante la etapa posterior a la floración, lo que provoca la eventual disminución del potencial de rendimiento, en particular cuanto mayor es la latitud y más se retrasa la siembra (Cirilo y Andrade, 1994).

La densidad de siembra (DS) óptima de un cultivo es aquella que maximiza el rendimiento en grano para una determinada asignación de recursos ambientales y tecnológicos. En maíz, el rendimiento en grano por unidad de área presenta una respuesta parabólica a la densidad de siembra (Willey y Heath, 1969), por lo que el máximo de la función determina el valor óptimo de la población (Sangoi, Gracietti, Rampazzo y Bianchetti, 2002; Li etal., 2015). Mientras el aumento gradual del número de plantas genera incrementos de la biomasa total al cubrir antes el suelo y utilizar mejor la energía lumínica, particularmente alrededor de la floración (Andrade, Otegui y Vega, 2000; Cantarero, Luque y Rubiolo, 2000), con el aumento de DS la mayor competencia por los recursos disponibles tiende a estabilizar la producción por unidad de superficie. 
Para cada planta individualmente, por su parte, el crecimiento muestra una disminución logarítmica a medida que la población aumenta (Li et al., 2015).

Una práctica que ha acompañado la decisión de siembra tardía en la región central, para ajustar mejor la necesidad del cultivo con la menor dotación de recursos disponibles, es reducir el stand de plantas de modo que $6 \mathrm{pl} \mathrm{m}^{-2}$ constituye una población habitual en esta época (Cirilo y Andrade, 1994; Cantarero, Luque y Rubiolo, 2000). Además de la influencia de la densidad de siembra sobre la productividad del maíz (Cox, 1996; Sarlangue, Andrade, Calviño y Purcell, 2007), uno de los parámetros que la población de plantas modifica a lo largo del ciclo es la cobertura del cultivo (de la Casa et al., 2016). Por otra parte, tanto el ambiente como el genotipo modifican la DS óptima en maíz (Friedman, 2016; Cerliani, Esposito, Morla, Balboa y Naville, 2018), de modo que resulta de interés conocer en qué medida el cambio de la densidad influye sobre el nivel productivo y la condición de riesgo.

La evaluación de RP es difícil de realizar experimentalmente debido a la necesidad de controlar de una manera integrada y efectiva los múltiples factores que limitan la productividad (Grassini, Thorburn, Burr y Cassman, 2011). Una forma alternativa de evaluar BR es mediante el uso de modelos de simulación de cultivos, que son herramientas de cálculo confiables cuando se dispone de información meteorológica y edáfica apropiada en extensión y detalle (Lobell et al., 2009). Por otra parte, los modelos de cultivos no deben ser necesariamente complejos a fin de garantizar estimaciones precisas del comportamiento productivo (Constantin, Willaume, Murgue, Lacroix y Therond, 2015). En este sentido, Battisti, Sentelhas y Boote (2017) recomiendan utilizar los resultados que producen diferentes modelos de cultivo de manera ensamblada a fin de producir estimaciones de productividad más consistentes y confiables, en especial cuando las condiciones ambientales son más extremas.

El modelo de simulación de cultivos AquaCrop (Raes, Steduto, Hsiao y Fereres, 2009; Steduto, Hsiao, Raes y Fereres, 2009), haciendo uso de la cobertura del cultivo (CC) como parámetro central para estimar la biomasa y la tasa de transpiración, ha demostrado un adecuado potencial como herramienta predictiva en la región central de la Argentina, tanto de la dinámica hídrica como del rendimiento del cultivo de maíz (de la Casa et al., 2016). AquaCrop se ha empleado extensivamente para analizar el comportamiento productivo de distintos cultivos bajo condiciones de manejo muy diversas. Hsiao et al. (2009) realizaron una parametrización y calibración inicial de AquaCrop para maíz, en tanto que Heng, Hsiao, Evett, Howell y Steduto (2009) lo aplicaron para evaluar el comportamiento de este cultivo en secano y bajo riego. Por su parte, Abedinpour et al. (2012) lo aplicaron para evaluar tres niveles de fertilización nitrogenada y cuatro de riego; Stricevic, Cosic, Djurovic, Pejic, y Maksimovic (2011) lo usaron en Serbia bajo dos escenarios (en secano y bajo riego, con suministro adecuado de nutrientes) evaluando los cultivos de maíz, remolacha y girasol. También para maíz, Flores-Gallardo, Ojeda-Bustamante, Flores-Magdaleno, Sifuentes-lbarra y MejíaSaénz (2013) emplearon AquaCrop en el norte de Sinaloa, México, para evaluar tres condiciones de disponibilidad hídrica: riego total (RT) y riego deficitario (80 \% y $60 \%$ con respecto a RT).

Desde su aparición, la plataforma de cálculo de AquaCrop ha incorporado un conjunto de rutinas que lo hacen muy apropiado a la hora de seleccionar un modelo para estimar la BR. Entre las mejoras que el modelo ha incorporado se destacan tres aspectos en particular. En primer lugar, mientras el valor de la evapotranspiración de referencia (ETo) obtenido a partir del procedimiento de Penman-Monteith (PM) se debía introducir inicialmente como un archivo de datos externo, su cálculo ahora es factible utilizando sólo los valores de temperatura máxima y mínima diarios que ingresan como parte de la información climática. En segundo lugar, presenta una modalidad de corridas sucesivas o secuenciales, lo que permite simular el comportamiento productivo de un cultivo a lo largo del tiempo y, de esta forma, ponderar de manera más fidedigna la humedad de suelo inicial de cada ciclo productivo considerando los aportes y el consumo de agua desde la cosecha del cultivo anterior. Por último, el modelo ha incorporado un módulo destinado a realizar la evaluación estadística de la simulación a partir de distintas medidas de ajuste y del error de estimación, permitiendo hacer un contraste inmediato entre los valores observados y estimados de CC, biomasa y agua del suelo.

En razón de que AquaCrop estima la productividad sobre la base de la eficiencia en el uso del agua, el empleo confiable de la herramienta exige la determinación adecuada del agua disponible para el cultivo. En tal sentido, la verosimilitud del modelo usado para representar la continuidad del agua en el sistema suelo-plantaatmósfera sólo puede sostenerse a partir de una rigurosa cuantificación tanto de la entrada como de la salida de agua. Por el lado del ingreso, la relación entre infiltración y escurrimiento, la distribución del agua en el perfil y la tasa efectiva 
de consumo son algunos aspectos fundamentales que el balance de agua de AquaCrop representa de manera apropiada (Raes et al., 2009), en particular cuando se utiliza un valor adecuado de curva número $(\mathrm{CN})$ para estimar el escurrimiento (de la Casa et al., 2016; 2018).

Con relación a la salida de agua, esta representación exhaustiva de la dinámica hídrica reduce su potencial si la tasa de demanda atmosférica no está bien ajustada para la región y estación de crecimiento. De esta forma, la determinación precisa de la tasa de evapotranspiración de referencia (ETo), a partir de la cual se estima el consumo máximo del cultivo en ausencia de estrés, resulta fundamental para evaluar de manera correcta la disponibilidad del agua. El modelo de PenmanMonteith (PM) implementado de acuerdo a las pautas de cálculo de FAO (Allen, Pereira, Raes y Smith, 1998), constituye el método más robusto y con mayor base física para estimar ETo (Irmak, Kabenge, Skaggs y Mutiibwa, 2012; McVicar et al., 2012). El valor de ETo que se obtiene del método de PM a partir del set completo de cuatro variables atmosféricas (radiación solar/heliofanía, temperatura y humedad del aire y velocidad del viento) constituye en la actualidad el procedimiento estándar para representar el poder evaporante de la atmósfera (Donohue, McVicar y Roderick, 2010; de la Casa y Ovando, 2016; Vicente-Serrano et al., 2017). Sin embargo, en muchos lugares del mundo la información meteorológica resulta aún incompleta para utilizar este procedimiento directamente, de modo que su empleo apelando a ciertas simplificaciones es un tema recurrente (Djaman et al., 2016). Almorox, Senatore, Quej y Mendicino (2016) proponen, en este sentido, obtener ETo siguiendo el método PM basado sólo en los registros de temperatura máxima y mínima.

El objetivo principal de este trabajo fue evaluar la capacidad del modelo AquaCrop para representar el comportamiento productivo del cultivo de maíz en secano de siembra tardía en la región central de Córdoba, Argentina y, bajo la modalidad de corridas sucesivas del modelo, estimar la dinámica temporal de la brecha de rendimiento a fin de establecer el nivel de riesgo de esta época de siembra más retrasada en la región. Los objetivos secundarios fueron: evaluar la rutina de cálculo de ETo diaria obtenida con el método PenmanMonteith cuando el cálculo utiliza sólo valores de temperatura máxima y mínima en lugar del set completo de variables meteorológicas que el procedimiento emplea en su versión original, con la finalidad de disponer una serie de tiempo de ETo más extendida para la región; analizar la influencia de distintas oportunidades de siembra tardía (fechas fijas y condicionadas a la ocurrencia de Iluvia previa) sobre la brecha productiva; evaluar la influencia de la densidad de siembra (6 u 8 pl $\mathrm{m}^{-2}$ ) sobre la condición de riesgo climático y su influencia en el rendimiento de indiferencia de la siembra tardía de maíz.

\section{MATERIALES Y MÉTODOS}

\section{Ubicación y datos del ensayo}

Los datos para calibrar el modelo AquaCrop se obtuvieron de un cultivo de maíz (Zea mays L.) de la variedad Dow 510PW/RR, en secano y fertilizado con nitrógeno, a partir de datos relevados entre siembra (17/12/2015) y madurez fisiológica (30/04/2016) en un lote de 45 ha ubicado al sur de la ciudad de Córdoba (313 $33^{\prime} \mathrm{S}, 64^{\circ} 05^{\prime} \mathrm{O}$; 400 m s.n.m.), como muestra la Figura 1. El suelo del predio es un Haplustol éntico, serie Manfredi, con un perfil de tres horizontes de textura franco-limosa, con cuyas características se elaboró el archivo de suelo que requiere AquaCrop. La información edáfica de los distintos horizontes se presenta en la Tabla 1.

Sobre el lote de forma rectangular se estableció una grilla de muestreo de 16 estaciones, 4 en sentido Norte-Sur por 4 en dirección Este-Oeste, en posiciones aproximadamente equidistantes. La calibración de AquaCrop se efectuó seleccionando de los ocho sectores monitoreados de manera intensiva, aquel que expresó mejor la variación del conjunto de mediciones en cuanto a materia seca (MS), agua del suelo (AS) y cobertura del cultivo (CC).

La densidad de siembra en la etapa de calibración del modelo fue de $6 \mathrm{pl} \mathrm{m}^{-2}$, valor que se verificó a partir del conteo de la cantidad de plantas en 5 metros lineales de dos filas contiguas. En cada fecha de muestreo se realizó el corte de la parte aérea de tres plantas para determinar la materia seca. El contenido de AS se determinó utilizando el método gravimétrico, a partir de un sondeo del perfil que permitió extraer muestras a cinco profundidades: 0,$1 ; 0,3 ; 0,5 ; 0,7$ y $0,9 \mathrm{~m}$. Estas mediciones se integraron a los efectos de obtener el contenido de agua del perfil hasta 1,60 $\mathrm{m}$, considerando un valor uniforme de densidad aparente de 1,25 $\mathrm{Mg} \mathrm{m}^{-3}$.

Por su parte, el desarrollo de CC se monitoreó a partir de fotografías adquiridas a $3 \mathrm{~m}$ por encima del canopeo del maíz, utilizando una cámara Canon PowerShot SD780 IS que fue modificada para producir imágenes de NDVI (de la Casa et 

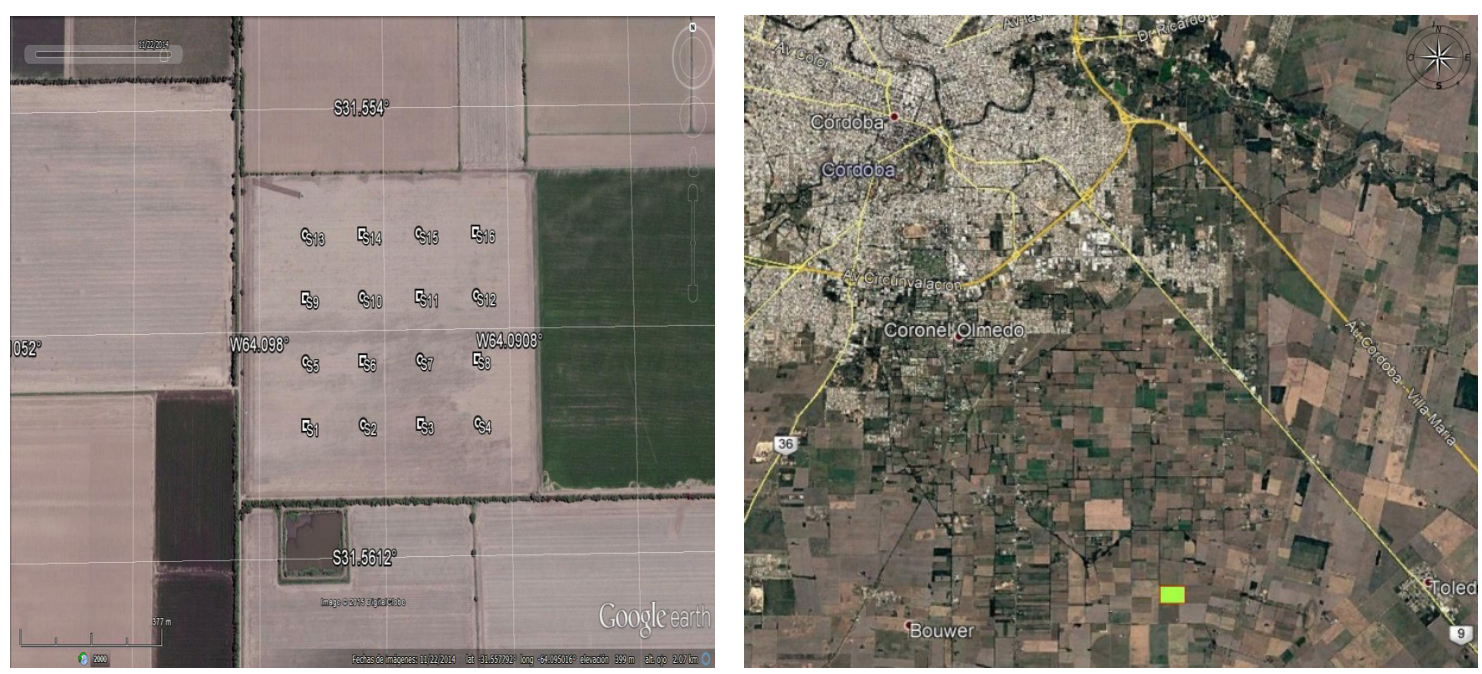

Figura 1. Imágenes de Google Earth con la ubicación geográfica del lote de maíz (rectángulo verde) al SE de la ciudad de Córdoba (izquierda), y su ampliación mostrando los 16 sectores de muestreo (derecha). Los símbolos cuadrados señalan sectores de muestreo más intensivo, cuyos datos fueron utilizados para calibrar AquaCrop.

Tabla 1. Propiedades edáficas utilizadas para representar el suelo del lote al sur de la ciudad de Córdoba, Argentina (Dardanelli, Bachmeier, Sereno y Gil, 1997).

\begin{tabular}{|c|c|c|c|c|c|c|c|c|}
\hline $\mathrm{Hz}$ & Textura & Profundidad & Saturación & CC & PMP & ADT & Ksat & CD \\
\hline & & $(\mathrm{m})$ & & $\left(m^{3} m^{-3}\right)$ & & $\left(\mathrm{mm} \mathrm{m}^{-1}\right)$ & $\left(m m\right.$ día $\left.^{-1}\right)$ & \\
\hline A & Franco & $0-0,23$ & 0,460 & 0,327 & 0,145 & 182 & 250 & 0,60 \\
\hline$A C$ & $\begin{array}{c}\text { Franco- } \\
\text { limoso }\end{array}$ & $0,24-0,46$ & 0,460 & 0,290 & 0,117 & 173 & 150 & 0,50 \\
\hline C & Limoso & $>0,47$ & 0,430 & 0,270 & 0,115 & 155 & 50 & 0,34 \\
\hline
\end{tabular}

Coeficiente de saturación; CD: Coeficiente de drenaje.

al., 2016; 2018). La obtención de CC se efectuó para cada imagen diferenciando el suelo de la vegetación verde por medio de un algoritmo de clasificación de máxima verosimilitud y, a partir de la imagen binaria producida, realizando el cálculo de la relación entre la cantidad de píxeles identificados como vegetación con respecto al total.

\section{Calibración de AquaCrop}

El modelo AquaCrop simula el desarrollo de CC y el resto de los procesos de crecimiento y desarrollo en una escala temporal que puede emplear tanto los días calendarios como el concepto de tiempo térmico. En virtud de que la simulación se extiende durante un lapso de tiempo prolongado sujeto a distintas condiciones de temperatura que pueden alterar la duración del ciclo, en esta oportunidad se implementó el segundo criterio para expresar la dinámica fenológica. Mientras que para la temperatura base se estableció un valor más generalizado en maíz de $10{ }^{\circ} \mathrm{C}$ (Gilmore y Rogers,
1958; Cross y Zuber, 1972; McMaster y Wilhelm, 1997), superior a $8{ }^{\circ} \mathrm{C}$ que utilizan Heng et al. (2009) y Hsiao et al. (2009), el límite superior se fijó en $30{ }^{\circ} \mathrm{C}$ y coincide con el valor que utilizan todos los autores mencionados.

El valor de productividad del agua (PA) utilizado para evaluar el modelo fue $33,2 \mathrm{~g} \mathrm{~m}^{-2}$, ligeramente inferior al de $33,7 \mathrm{~g} \mathrm{~m}^{-2}$ que emplearon de la Casa et al. (2016) para un cultivo de maíz bajo riego en el mismo lote. Estos valores de PA se encuentran próximos al límite superior del rango que AquaCrop propone para las especies de metabolismo C4, y tampoco difiere mayormente del valor utilizado por Heng et al. (2009) y Hsiao et al. (2009). Los valores de rendimiento estimados para la serie completa se ajustaron al valor de PA usado en la calibración para referir las estimaciones a la misma concentración atmosférica de $\mathrm{CO}_{2}$ (Steduto et al., 2009).

Calibrado para representar el cultivo de maíz de secano en el área central de Córdoba, el modelo AquaCrop se utilizó a modo de herramienta predictiva para analizar en primer término si, en un 
contexto de siembra tardía, existe una oportunidad que pueda resultar más conveniente. En tal sentido, AquaCrop se corrió de manera secuencial bajo un esquema de siembra que cada año considera, por un lado, tres fechas fijas (15 de noviembre, 1 de diciembre y 17 de diciembre) y, por el otro, una toma de decisión basada en la acumulación de agua previa que, en esta oportunidad, evaluó $50 \mathrm{~mm}, 100 \mathrm{~mm}$ y $150 \mathrm{~mm}$ de lluvia acumulados desde el 1 de noviembre.

A fin de considerar la influencia del aumento en la densidad de siembra sobre BR, se estimó la variación de CC a lo largo del ciclo para la densidad de $8 \mathrm{pl} \mathrm{m}^{-2}$, siguiendo el conjunto de coeficientes de desarrollo del canopeo obtenidos por de la Casa et al. (2016) para la misma región, de acuerdo a los valores que se presentan en la Tabla 2.

Tabla 2. Coeficientes del modelo de desarrollo de la cobertura de maíz que utiliza AquaCrop para representar las dos densidades de siembra.

\begin{tabular}{|c|c|c|c|c|}
\hline Densidad & $\mathrm{CC}_{0}$ & CCx & CGC & CDC \\
\hline pl $\mathbf{m}^{-2}$ & \multicolumn{2}{|c|}{$\%$} & \multicolumn{2}{|c|}{$\%\left({ }^{\circ} \mathrm{C} \mathrm{d}\right)^{-1}$} \\
\hline 6 & 0,39 & 89 & 1,39 & 1,30 \\
\hline 8 & 0,52 & 99 & 1,37 & 1,30 \\
\hline
\end{tabular}

Referencias: $\mathrm{CC}_{0}$ : tamaño inicial del canopeo $(\mathrm{en} \mathrm{t}=0$ ) estimado de acuerdo a la densidad de plantas; CCx: cobertura máxima en condiciones óptimas de crecimiento; CGC: coeficiente de crecimiento del canopeo; CDC: coeficiente de declinación del canopeo.

El modelo calibrado para $6 \mathrm{pl} \mathrm{m}^{-2} \mathrm{y}$ tomando el 17 de diciembre como fecha de siembra se corrió de manera sucesiva o secuencial entre 1960 y 2017, tanto en condiciones de secano para estimar el rendimiento de maíz de carácter real (RR), como sin limitaciones hídricas a fin de obtener el correspondiente valor potencial (RP).

\section{Información meteorológica y cálculo de ETo}

La estimación de BR en maíz a lo largo del tiempo a partir de AquaCrop exige disponer de una base de datos meteorológicos prolongada o, en su defecto, evaluar la magnitud del error sujeto el cálculo de ETo cuando se emplea un procedimiento alternativo o no se utiliza el conjunto completo de cuatro variables fundamentales. En este sentido, la dotación mínima de datos meteorológicos que se requiere para calcular ETo siguiendo el método PM son los registros de temperatura máxima (Tx) y mínima ( $T n$ ) (FAO, 2012; Almorox et al., 2016), cuya disponibilidad en tiempo y espacio es más generalizada. Otro aspecto de interés a considerar es la distancia que separa la estación de procedencia de los datos meteorológicos respecto del área de aplicación de la información producida.

Los datos meteorológicos utilizados en el estudio proceden de dos fuentes diferentes: una estación automática (EA) ubicada próxima al lote

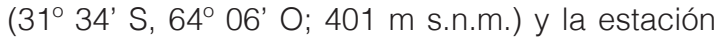
Córdoba Observatorio (BO) que se encuentra en la ciudad de Córdoba y depende del Servicio Meteorológico Nacional (31 24' S, 64 11' O; 425 m s.n.m.). La distancia que separa a estas estaciones meteorológicas es de $17 \mathrm{~km}$. La calibración del modelo en 2015-2016 se realizó utilizando los registros de la estación automática que, además de la lluvia, registra el set completo de variables meteorológicas empleadas para calcular la tasa diaria de evapotranspiración de referencia por el método de Penman-Monteith (ETo_PM) (Allen et al., 1998). Como esta estación fue instalada a fines de 2010, existe información meteorológica desde entonces que se utilizó para calcular ETo_ PM a partir del set completo de cuatro variables fundamentales. Con los valores de temperatura máxima (Tx) y mínima (Tn) de esta estación también se obtuvo ETo (ETo_PM ${ }_{\text {TXTn }}$ ), de acuerdo a la rutina de cálculo que utiliza el programa ETo_Calculator versión 3.1 (FAO, 2012).

Por su parte, la estación BO proveyó los registros diarios de Tx, Tn y lluvia entre 1960 y 2017. A partir de Tx y Tn se aplicó el mismo procedimiento anterior para estimar diariamente ETo_PM $_{\text {TXTn }}$. Los valores de ETo estimados a partir de Tx y Tn en ambos casos fueron analizados por correlación y regresión con los valores de ETo calculados con el método PM estándar a partir del conjunto completo de variables meteorológicas de la estación automática. Si bien se presentan algunas discontinuidades por mantenimiento del equipo y cortes eléctricos, la serie abarca el lapso extendido entre 2011 y 2017. El análisis se realizó considerando el conjunto completo de la información disponible ( $n=1667$ días), como así también para cada año en particular a fin de conocer el rango de variación.

\section{Evaluación estadística}

Los valores de ETo calculados con PM a partir de las cuatro variables fundamentales y las respectivas estimaciones se evaluaron por correlación y regresión, en tanto el coeficiente de determinación $\left(R^{2}\right)$ y la raíz cuadrada del error cuadrático medio (RMSE) se utilizaron para analizar la bondad del ajuste. 
Empleando la rutina de cálculo que dispone la propia plataforma, la calibración de AquaCrop se implementó analizando las diferencias a lo largo del ciclo entre valores observados y estimados de la cobertura del cultivo (CC), la materia seca aérea acumulada (MS) y el contenido de agua del suelo (AS). Para el conjunto de valores observados en cada sector de muestreo, el criterio adoptado para realizar la calibración se basó en seleccionar los parámetros obtenidos en aquel sector con el mayor estándar de ajuste y error de estimación más bajo. A tal efecto se utilizaron los distintos indicadores que el modelo determina, esto es: coeficiente de correlación (r), raíz cuadrada del error cuadrático medio (RMSE), raíz cuadrada del error cuadrático medio normalizada (NRMSE), coeficiente de eficiencia del modelo de Nash-Sutcliffe (EF) e índice de concordancia de Willmott (d) (Willmott, Robeson y Matsuura, 2012).

La prueba de $t$ para la diferencia de medias de muestras apareadas se utilizó a los efectos de establecer la significación estadística, tanto de las fechas de siembra como de los tratamientos de densidad que fueron evaluados para $p<0,05$.

\section{RESULTADOS Y DISCUSIÓN}

\section{Estimación de la evapotranspiración de referencia a partir de Tx y Tn (ETo_PM $\mathrm{TxTn}_{\mathrm{T}}$ )}

Para evaluar las diferencias entre los valores de ETo obtenidos a partir de PM con el set completo de información meteorológica y de aquellos calculados sólo a partir de Tx y Tn, se estableció la relación lineal entre ambos. La Figura 2 presenta los resultados del análisis de correlación y regresión entre valores diarios de ETo que se obtienen a partir del set completo de cuatro variables fundamentales (ETo_PM) en abscisa y los estimados utilizando sólo Tx y Tn como información meteorológica (ETo_PM $\mathrm{T}_{\text {TXTn }}$ ) en ordenada. En este caso, se hizo uso tanto de los registros de la estación automática (EA) como de la convencional localizada en la ciudad de Córdoba (BO), a fin de analizar la influencia de la distancia que separa la fuente de datos meteorológicos del área de aplicación. En la Tabla 3 se muestran los parámetros de la recta de regresión entre los respectivos valores de ETo, como así también las medidas de ajuste $\left(R^{2}\right)$ y error (RMSE) para cada año civil por separado y el conjunto completo de la información disponible, a fin de mostrar su variabilidad.

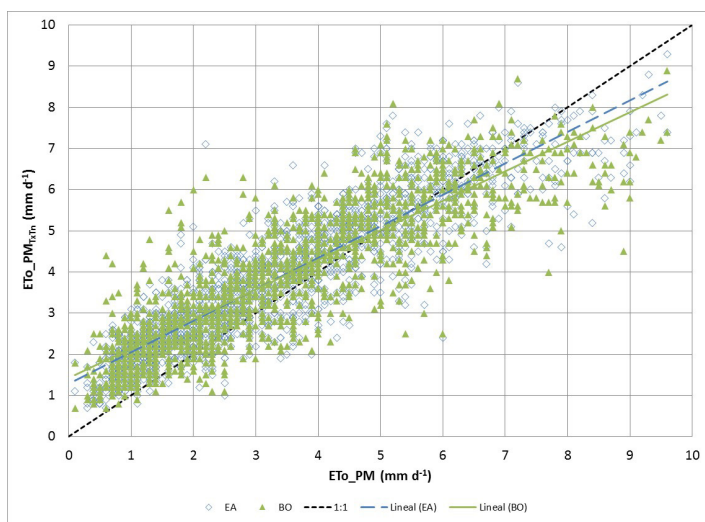

Figura 2. Relación entre los valores diarios de evapotranspiración de referencia obtenida con el método de Penman-Monteith utilizando las cuatro variables fundamentales (ETo_PM) y la calculada utilizando sólo la temperatura máxima y mínima (ETo_ $\mathrm{PM}_{\mathrm{TXT}}$ ), para los datos in situ de la estación automática (EA) y de la estación convencional en la ciudad de Córdoba (BO).

La Figura 2 muestra que el modelo simplificado de PM que utiliza sólo Tx y Tn para calcular ETo produce estimaciones semejantes a ETo_PMa partir del set completo de información meteorológica, si bien sobrestima los valores más bajos y subestima los más elevados. Además, este comportamiento se

Tabla 3. Coeficientes de regresión (a: ordenada al origen; b: pendiente), de determinación (R2) y raíz media del error cuadrático (RMSE; mm d-1) de la relación lineal entre los valores diarios de evapotranspiración de referencia obtenida con el método de Penman-Monteith utilizando las cuatro variables fundamentales (ETo_PM) y la calculada utilizando sólo temperatura máxima y mínima (ETo_PMTXTn), para los datos de la estación automática (EA) y de la estación convencional en la ciudad de Córdoba (BO) considerando años individuales y el conjunto completo.

\begin{tabular}{lcccccccccccc}
\hline Año & \multicolumn{2}{c}{2011} & \multicolumn{2}{c}{2012} & \multicolumn{2}{c}{2013} & \multicolumn{2}{c}{2014} & \multicolumn{2}{c}{2015} & \multicolumn{2}{c}{$2011-2015$} \\
n & \multicolumn{2}{c}{360} & \multicolumn{2}{c}{331} & \multicolumn{2}{c}{329} & \multicolumn{2}{c}{315} & & 332 & \multicolumn{2}{c}{1667} \\
Estación & EA & BO & EA & BO & EA & BO & EA & BO & EA & BO & EA & BO \\
\hline a & 1,094 & 1,270 & 1,171 & 1,315 & 1,392 & 1,630 & 1,365 & 1,396 & 1,465 & 1,523 & 1,293 & 1,423 \\
b & 0,815 & 0,766 & 0,805 & 0,743 & 0,748 & 0,682 & 0,775 & 0,737 & 0,672 & 0,656 & 0,764 & 0,717 \\
R $^{2}$ & 0,792 & 0,732 & 0,809 & 0,794 & 0,770 & 0,719 & 0,865 & 0,810 & 0,767 & 0,716 & 0,798 & 0,753 \\
RMSE & 0,954 & 1,058 & 0,999 & 1,001 & 1,102 & 1,198 & 1,027 & 1,091 & 1,005 & 1,084 & 1,017 & 1,088 \\
\hline
\end{tabular}


exacerba (disminuye la pendiente) en la medida en que la procedencia de los datos de Tx y Tn es más distante, como cuando se emplean los registros de la ciudad de Córdoba (BO). No obstante, analizando los distintos años por separado, los coeficientes de la Tabla 3 muestran para EA que el rango de $R^{2}$ está comprendido entre 0,77 y 0,86 y el de RMSE entre 0,95 y $1,10 \mathrm{~mm} \mathrm{~d}^{-1}$, en tanto que con respecto a $\mathrm{BO}$ los valores de $\mathrm{R}^{2}$ se reducen $(0,71$ y 0,81$)$, y los de RMSE se incrementan $(1,0$ y $\left.1,2 \mathrm{~mm} \mathrm{~d}^{-1}\right)$, de acuerdo al año considerado. Estos valores confirman los resultados de Almorox et al. (2016), quienes obtuvieron un $\mathrm{R}^{2}$ próximo a 0,8 para los climas templados de la clasificación de Koeppen cuando analizan distintos modelos más simplificados para calcular ETo respecto al método estándar (PM).

\section{Calibración del modelo AquaCrop para maíz tardío en secano}

El cultivo de maíz sembrado a mediados de diciembre de 2015 alcanzó rendimientos elevados y muy uniformes en el lote monitoreado, de acuerdo tanto a la información del mapa de rendimiento $\left(12,1+/-1,1\right.$ t ha ${ }^{-1}$ para $\left.n=31227\right)$ como a las estimaciones realizadas en ocho sectores de muestreo $\left(13,4+/-1,0\right.$ t ha $\left.{ }^{-1}\right)$. Esta productividad es similar a los valores más altos que Grassini, Yang y Cassman (2009) presentan para los maíces de secano en los Estados Unidos de América y que, en ese caso, sólo son superados por los rendimientos obtenidos bajo riego.

La Figura 3 presenta los valores observados de CC, MS y AS a lo largo del ciclo y los estimados por AquaCrop correspondientes al sector 8, junto al diagrama de dispersión de los respectivos valores con respecto a la recta de identidad y la evaluación estadística resultante. Las medidas de ajuste y error obtenidas (Tabla 4) ponen de manifiesto una similitud consistente entre la verdad de campo y las estimaciones del modelo de manera simultánea
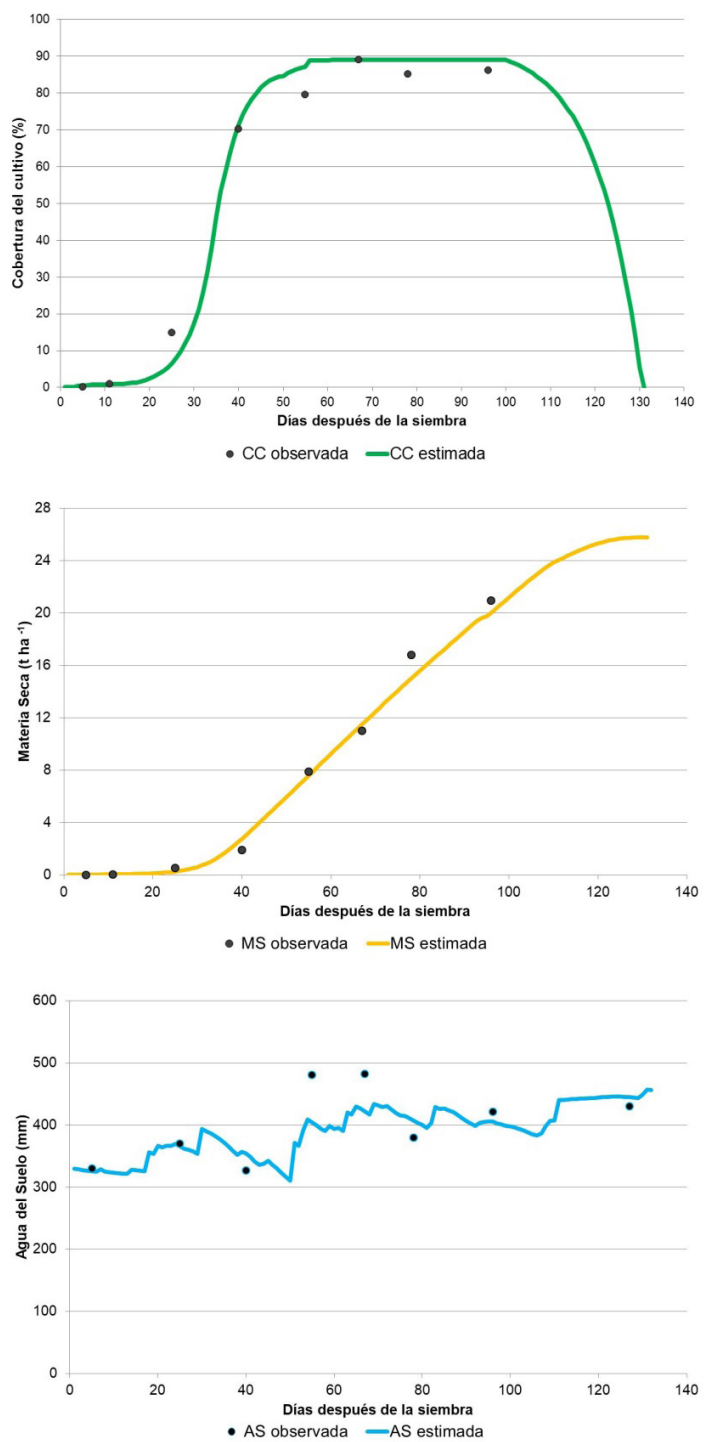

Figura 3. Valores de cobertura (CC), materia seca acumulada (MS) y agua del suelo (AS) observados en el sector 8 del lote y estimados por AquaCrop calibrado para el cultivo de maíz de siembra tardía cuyo ciclo transcurre entre diciembre de 2015 y abril de 2016 en Córdoba, Argentina.

Tabla 4. Valoración estadística de la relación entre valores observados de cobertura del cultivo, materia seca y contenido de agua del suelo hasta 1,6 m y estimados por AquaCrop en el sector 8 durante el ciclo 2015-2016 en Córdoba, Argentina.

\begin{tabular}{lccc}
\hline Parámetros estadísticos & Cobertura del cultivo $\mathbf{n}=\mathbf{8}$ & Materia seca $\mathbf{n}=\mathbf{9}$ & Agua del suelo $\mathbf{n}=\mathbf{8}$ \\
\hline $\mathbf{r}$ & 0,99 & 0,99 & 0,79 \\
EF & 0,99 & 0,98 & 0,56 \\
$\mathbf{d}$ & 0,99 & 0,99 & 0,82 \\
RMSE & $4,4 \%$ & $1,2 \mathrm{t} \mathrm{ha}$ & $38,0 \mathrm{~mm}$ \\
NRMSE & $8,2 \%$ & $12,5 \%$ & $9,4 \%$ \\
Media Observados & $53,2 \%$ & $9,73 \mathrm{t} \mathrm{ha}^{-1}$ & $402,4 \mathrm{~mm}$ \\
Media Estimados & $54,2 \%$ & $9,20 \mathrm{t} \mathrm{ha}^{-1}$ & $391,2 \mathrm{~mm}$ \\
\hline
\end{tabular}


para las tres variables, lo que avala su uso para simular el crecimiento y desarrollo de maíz tardío en esta región. La menor correlación observada en los otros sectores, particularmente en relación a AS, se justifica ya que cada sector de muestreo requiere ajustar la curva número de acuerdo a su ubicación en el terreno para estimar de manera apropiada el escurrimiento superficial de agua (de la Casa et al., 2016). En síntesis, la herramienta se considera apropiada a fin de extrapolar temporalmente y evaluar la condición de riesgo climático para el conjunto de los ciclos productivos.

Para una población de $6 \mathrm{pl} \mathrm{m}^{-2}$ el cultivo de maíz no alcanza cobertura completa $(C C x<100$ $\%)$, como se aprecia en la Figura 3, donde CCx presenta un valor de $89 \%$ para el sector 8 , mientras que el valor de CCx osciló entre $86 \%$ (sector 9 ) y $92 \%$ (sector 4) en el resto de sectores monitoreados.

Las estimaciones de rendimiento que produjo el modelo calibrado para $6 \mathrm{pl} \mathrm{m}^{-2}$ tomando como fecha de siembra el 17 de diciembre y ejecutado de manera secuencial entre 1960 y 2017 se presentan en la Figura 4, expresadas en términos de frecuencia relativa acumulada de RR a fin de cuantificar la probabilidad empírica. Durante todo el periodo analizado RP presentó valores entre 13 y $15 \mathrm{t} \mathrm{ha}^{-1}$, en tanto que el $65 \%$ de los años, RR superó las 13 tha ${ }^{-1}$

Por su parte, la Figura 6 muestra la BR en los mismos términos probabilísticos para el conjunto completo de fechas de siembra evaluadas. Sólo para la siembra de mediados de diciembre y asumiendo por completo la ausencia de pérdidas productivas por otras causas, el cultivo de maíz presenta reducciones nulas de la productividad en $30 \%$ de los ciclos, mientras que en $38 \%$ de ellos la pérdida resulta sólo ligera, asumiendo un valor arbitrario inferior a 2 t ha $^{-1}$ (aproximadamente el valor medio de BR es 2,1 $\mathrm{tha}^{-1}$ ). En el sentido opuesto, alrededor de $31 \%$ de los años las pérdidas de naturaleza climáticas producen disminuciones relativamente elevadas por encima de este umbral arbitrario $\left(2 \mathrm{t} \mathrm{ha}^{-1}\right)$.

\section{Brecha de rendimiento para maíz tardío en la región central de Córdoba, Argentina, considerando distintas oportunidades de siembra y una densidad de siembra de $6 \mathrm{pl} \mathrm{m}^{-2}$}

Los valores de BR para el conjunto de seis fechas de siembra evaluadas se analizaron

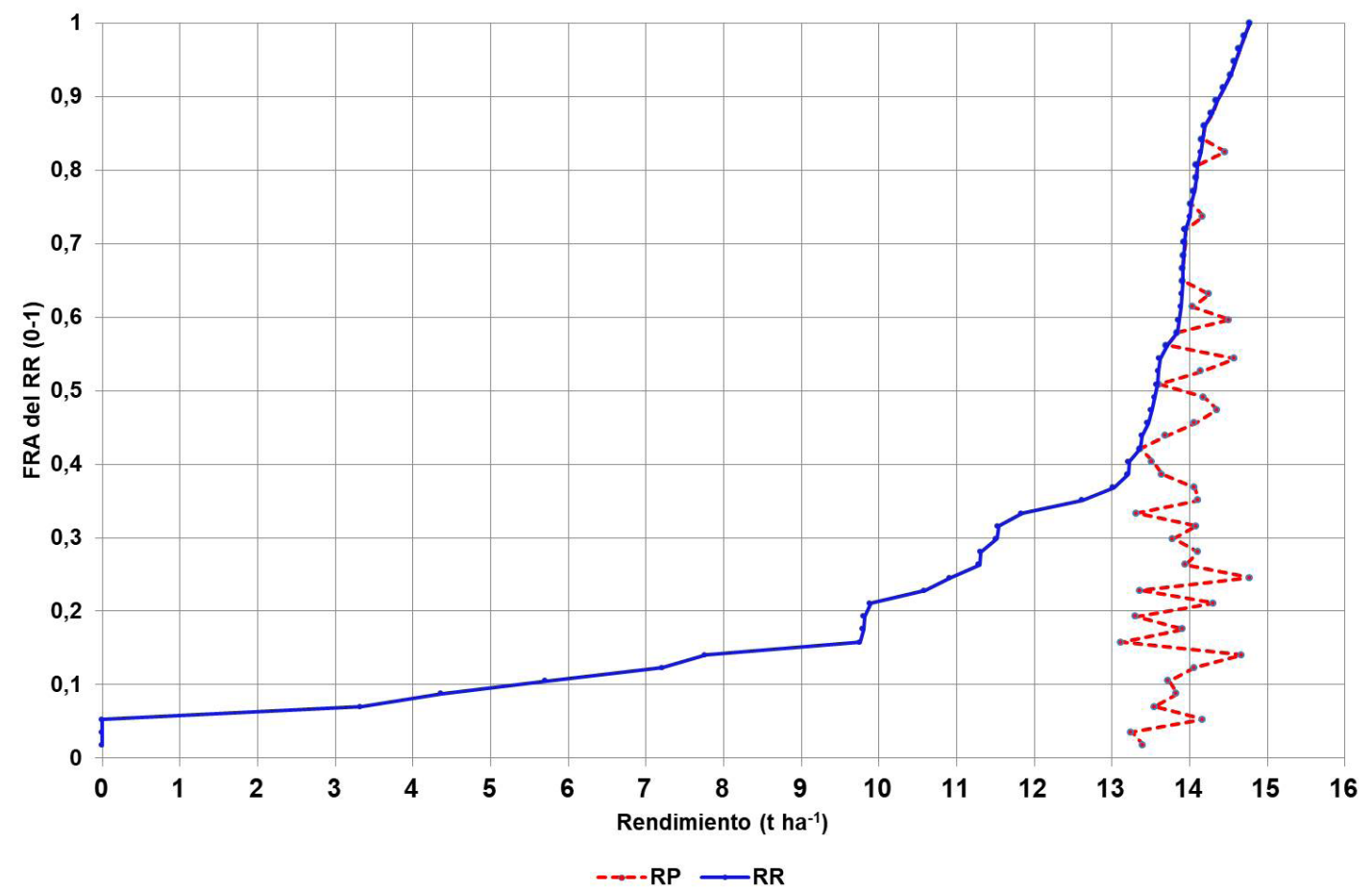

Figura 4. Frecuencia relativa acumulada (FRA) de los valores estimados de rendimiento real (RR) y el respectivo rendimiento potencial (RP) del año para el cultivo de maíz entre 1960 y 2017 ( $n=57$ años) en Córdoba, Argentina, con una densidad de 6 pl m-2 y fecha de siembra el 17 de diciembre. 


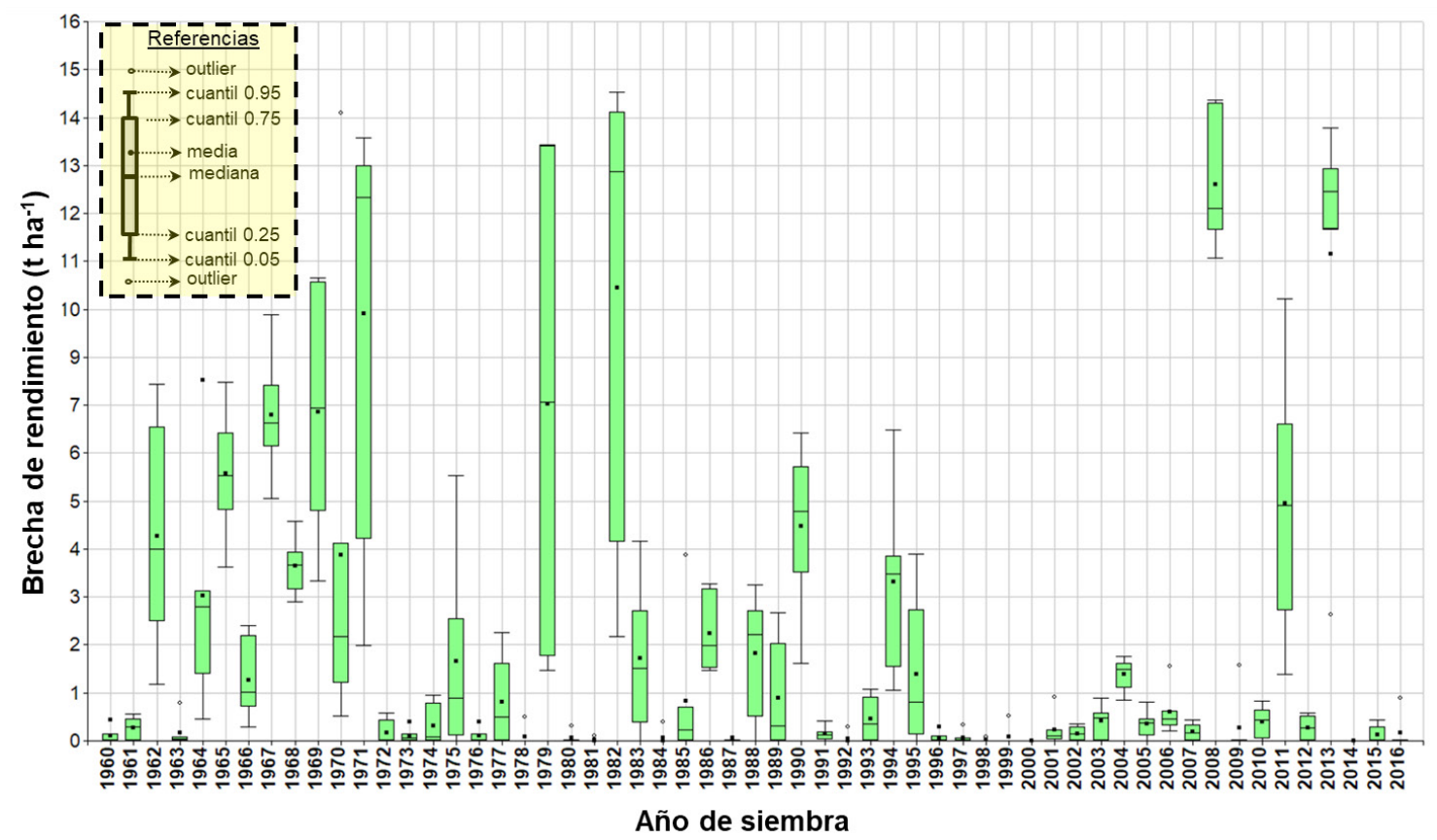

Figura 5. Variación de la brecha de rendimiento estimada para el cultivo de maíz de ciclo tardío sembrado entre 1960 y 2016 ( $n=57$ años) en la región central de Córdoba, Argentina, con una densidad de $6 \mathrm{pl} \mathrm{m} \mathrm{m}^{-2}$ y considerando seis oportunidades de siembra: fechas fijas todos los años: 15/11, 01/12 y 17/12 y fechas variables: cuando se acumulan $50 \mathrm{~mm}, 100 \mathrm{~mm}$ y $150 \mathrm{~mm}$ de lluvia desde 1 de noviembre. En el interior de la figura se muestran las referencias de los diagramas de cajas.

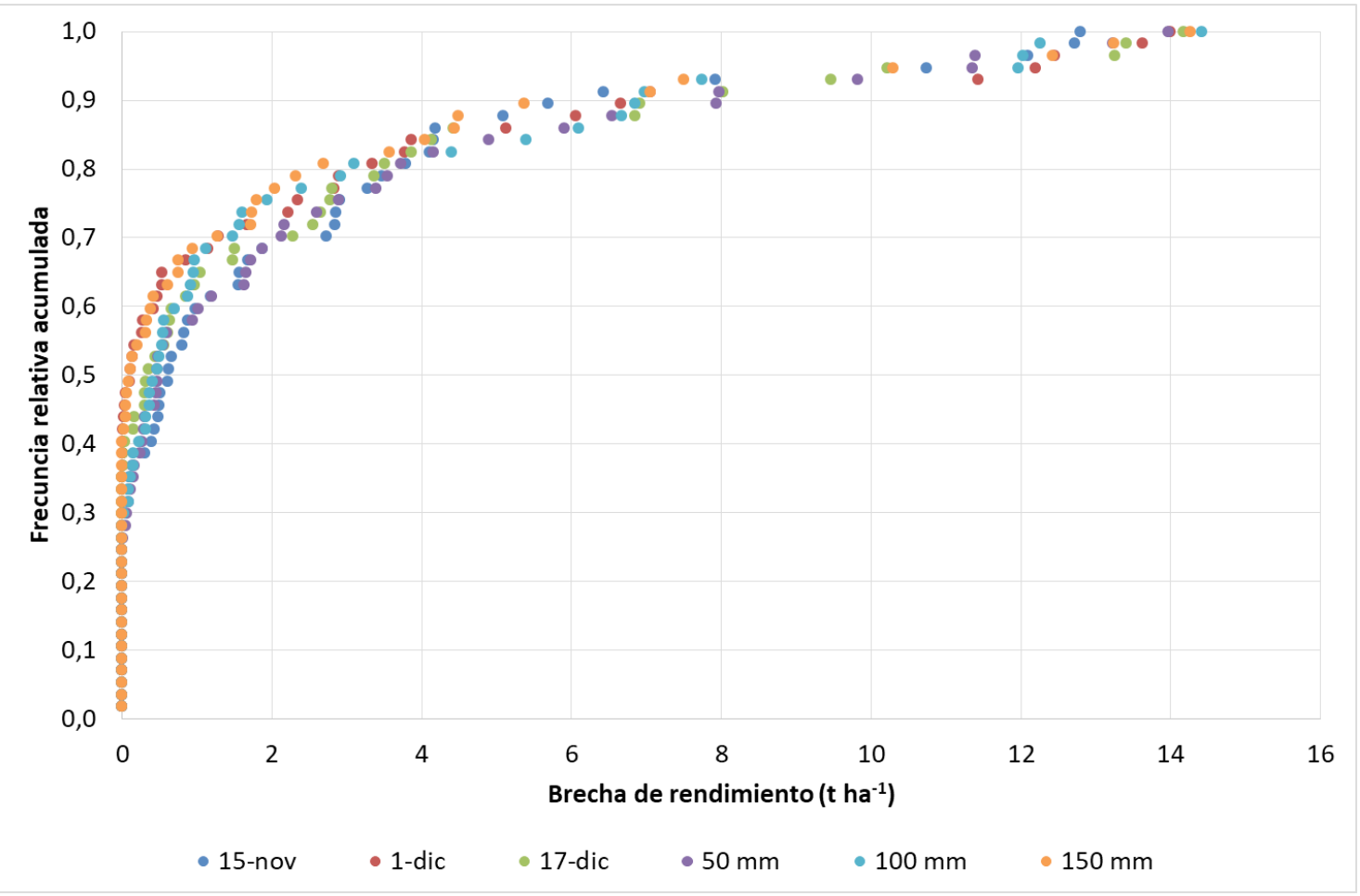

Figura 6. Frecuencia relativa acumulada de la brecha de rendimiento del cultivo de maíz estimada entre 1960 y 2017 ( $n=57$ años) en Córdoba, Argentina, para distintas fechas de siembra con una densidad de 6 pl m². 
estadísticamente cada año y estos resultados organizados en diagramas de caja se presentan en la Figura 5. Una lectura descriptiva de la Figura 5 permite separar años de BR reducidas y BR elevadas, es decir ciclos productivos de mayor y menor potencial climático, respectivamente. Los de menor potencial, relacionados a condiciones de sequía agrícola intensa y/o prolongada, corresponden a las campañas 1971-1972, 19821983, 2008-2009 y 2013-2014, con BR que superan $9 \mathrm{t} \mathrm{ha}^{-1}$. Por otra parte, mientras que en algunos ciclos productivos la oportunidad de siembra no tiene un impacto relevante sobre $\mathrm{BR}$, dando lugar a desvíos/rangos reducidos que expresan la indiferencia con respecto a la decisión de siembra cada año, en otros se producen diferencias ostensibles de BR bajo la influencia de la diferente oportunidad. Esto es que un pequeño adelanto o atraso de la siembra, modifica sustancialmente la productividad efectiva.

La prueba de $t$ para la diferencia de medias con muestras apareadas, como muestra la Tabla 4, no arrojó valores de probabilidad significativos $(p>0,10)$ entre las distintas fechas (fijas y variables), razón por la cual ninguna ocasión puede considerarse más apropiada que otra y pone de relieve el carácter azaroso de la decisión para este cultivo. Por su parte, la Figura 6 presenta el comportamiento probabilístico de todas las oportunidades de siembra tardía evaluadas. En general, existe $30 \%$ de probabilidad que BR presente una magnitud más sustancial (con valores mayores a $1,27 \mathrm{t} \mathrm{ha}^{-1}$ para la fecha determinada por una acumulación de $150 \mathrm{~mm}$ de lluvia y a 2,73 t ha-1 cuando la siembra se realiza todos los años el 15 de noviembre). Otra información relevante para la región es la pérdida de productividad muy acentuada (superior a 6,4 tha-1 para la siembra del 15 de noviembre y a 8,0 t ha $^{-1}$ cuando la siembra se realiza el 17 de diciembre) que se puede esperaren alrededor de $10 \%$ de los años (en 6 años de los 57 analizados), que son ocasionadas por los eventos de sequía más extremos.

\section{Brecha de rendimiento para maíz tardío en la región central de Córdoba, Argentina, considerando distintas oportunidades de siembra y $8 \mathrm{pl} \mathrm{m}^{-2}$}

La densidad de $6 \mathrm{pl} \mathrm{m} \mathrm{m}^{-2}$ puede considerarse una población sub-óptima en la medida que el cultivo no alcanza la cobertura completa $(C C x<100 \%)$ durante el ciclo y, en consecuencia, el maíz estaría resignando potencial de rendimiento en términos de una menor captura de luz y una tasa de

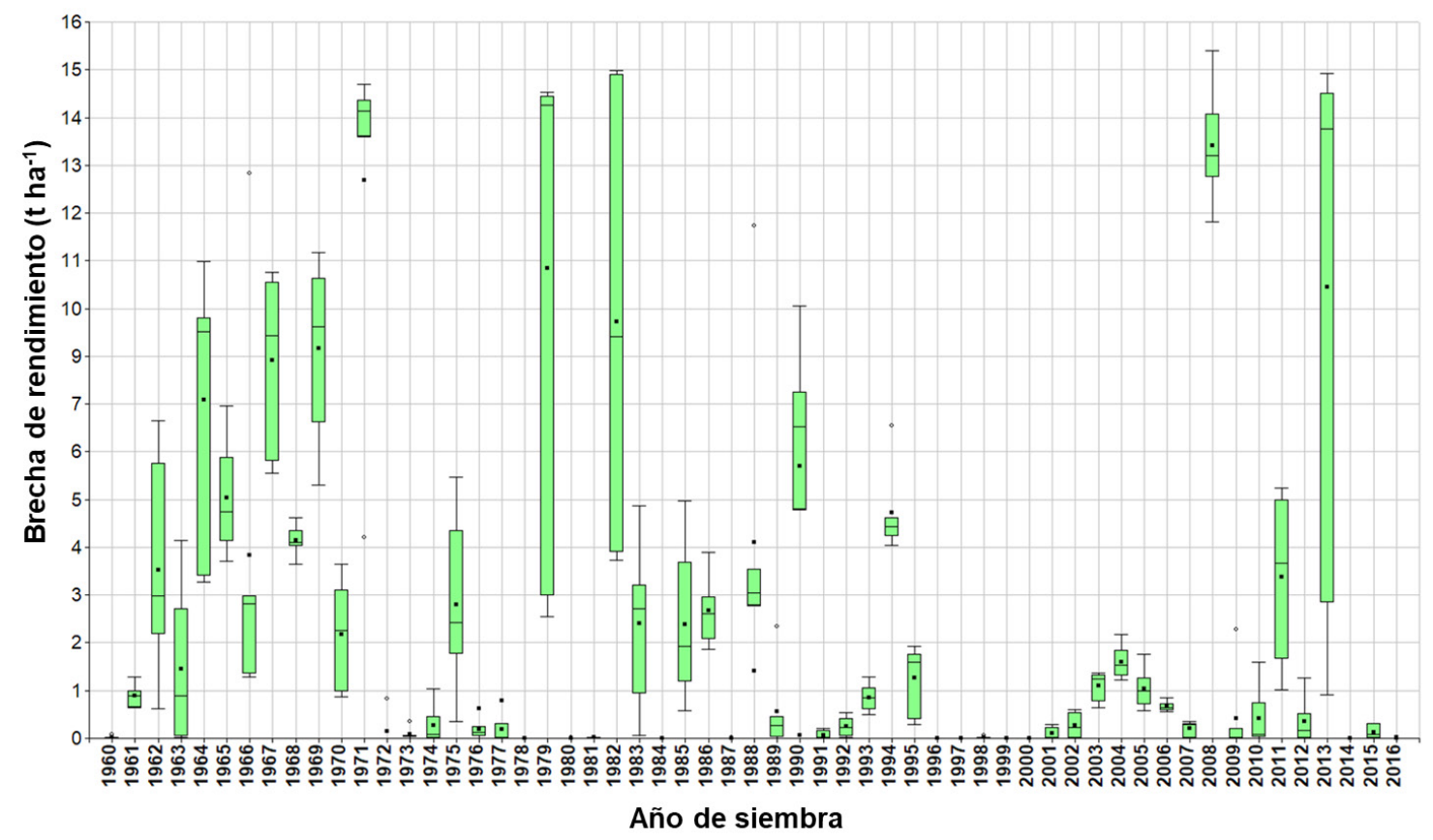

Figura 7. Variación de la brecha de rendimiento estimada para el cultivo de maíz de ciclo tardío sembrado entre 1960 y 2016 (n=57 años) en la región central de Córdoba, Argentina, con una densidad de 8 pl $\mathrm{m}^{-2}$ y considerando seis oportunidades de siembra: fechas fijas todos los años: 15/11, 01/12 y 17/12 y fechas variables: cuando se acumulan 50 mm, 100 mm y 150 mm de lluvia desde 1 de noviembre. Las referencias de los diagramas de caja son las mismas que en la Figura 5. 


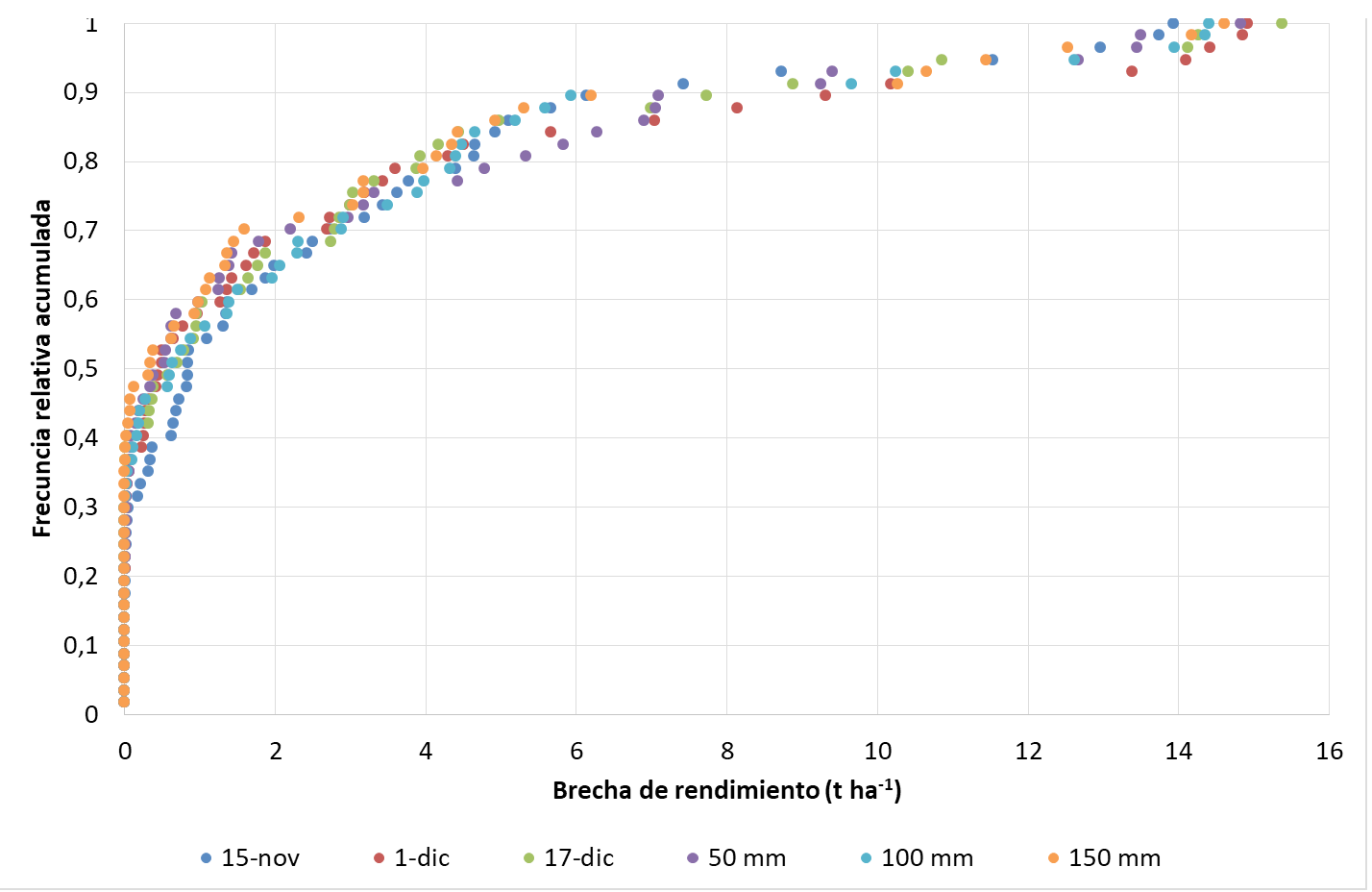

Figura 8. Frecuencia relativa acumulada de la brecha de rendimiento (BR) del cultivo de maíz estimada entre 1960 y 2017 ( $n=57$ años) en Córdoba, Argentina, considerando distintas fechas de siembra y una densidad de 8 pl m².

Tabla 5. Valores de correlación ( $r$ ) y de la prueba de $t$ para la diferencia de medias apareadas de la brecha de rendimiento entre distintas fechas de siembra tardía de maíz bajo dos densidades.

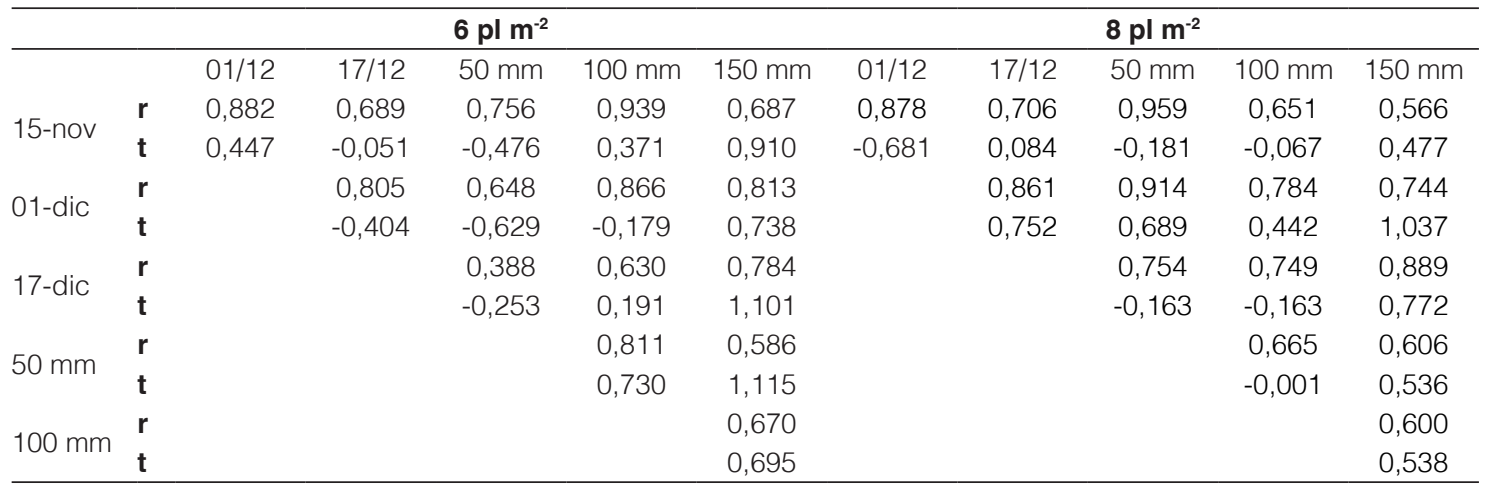

Todos los valores de r significativos $(\mathrm{P}<0,01)$ y los de t no significativos

evapotranspiración más reducida.

A los efectos de poner a prueba si el agua disponible es suficiente para sostener un incremento moderado de DS, se repitió el análisis de fechas de siembra precedente aumentando la densidad a $8 \mathrm{pl} \mathrm{m}^{-2}$. Este aumento de DS se asoció, en primer lugar, con un valor más elevado de CCo (tamaño inicial del canopeo en $\mathrm{t}=0$ ), el incremento de CCx, de modo que el cultivo de maíz presenta CC $x=99 \%$ y, además, un valor más alto de la tasa de desarrollo del canopeo (CGC) que permite aumentar el tamaño de la curva de cobertura (Steduto et al., 2009; de la Casa et al., 2016). Los resultados obtenidos se presentan en la Figura 7 por medio de diagramas de caja, en tanto la Figura 8 muestra las frecuencias relativas acumuladas para cada una de las fechas de siembra.

En este caso, como muestra la Tabla 5, tampoco se presentan valores de probabilidad significativos $(p>0,10)$ para la diferencia de medias apareadas entre las distintas fechas (fijas y variables) de modo que ninguna fecha tardía puede considerarse 
Tabla 6. Valores de correlación ( $r$ ), de la prueba de ty su probabilidad para la diferencia de medias apareadas de brechas de rendimiento entre distintas densidades de siembra.

\begin{tabular}{|c|c|c|c|c|c|c|}
\hline & \multicolumn{6}{|c|}{ Tratamientos } \\
\hline & $15 / 11$ & $01 / 12$ & $17 / 12$ & $50 \mathrm{~mm}$ & $100 \mathrm{~mm}$ & $150 \mathrm{~mm}$ \\
\hline $\mathbf{r}$ & 0,996 & 0,975 & 0,996 & 0,750 & 0,624 & 0,655 \\
\hline t & $-6,470$ & $-4,519$ & $-5,313$ & $-0,613$ & $-1,007$ & $-1,114$ \\
\hline$P(T<=t)$ & $<0,001$ & $<0,001$ & $<0,001$ & 0,542 & 0,318 & 0,270 \\
\hline
\end{tabular}

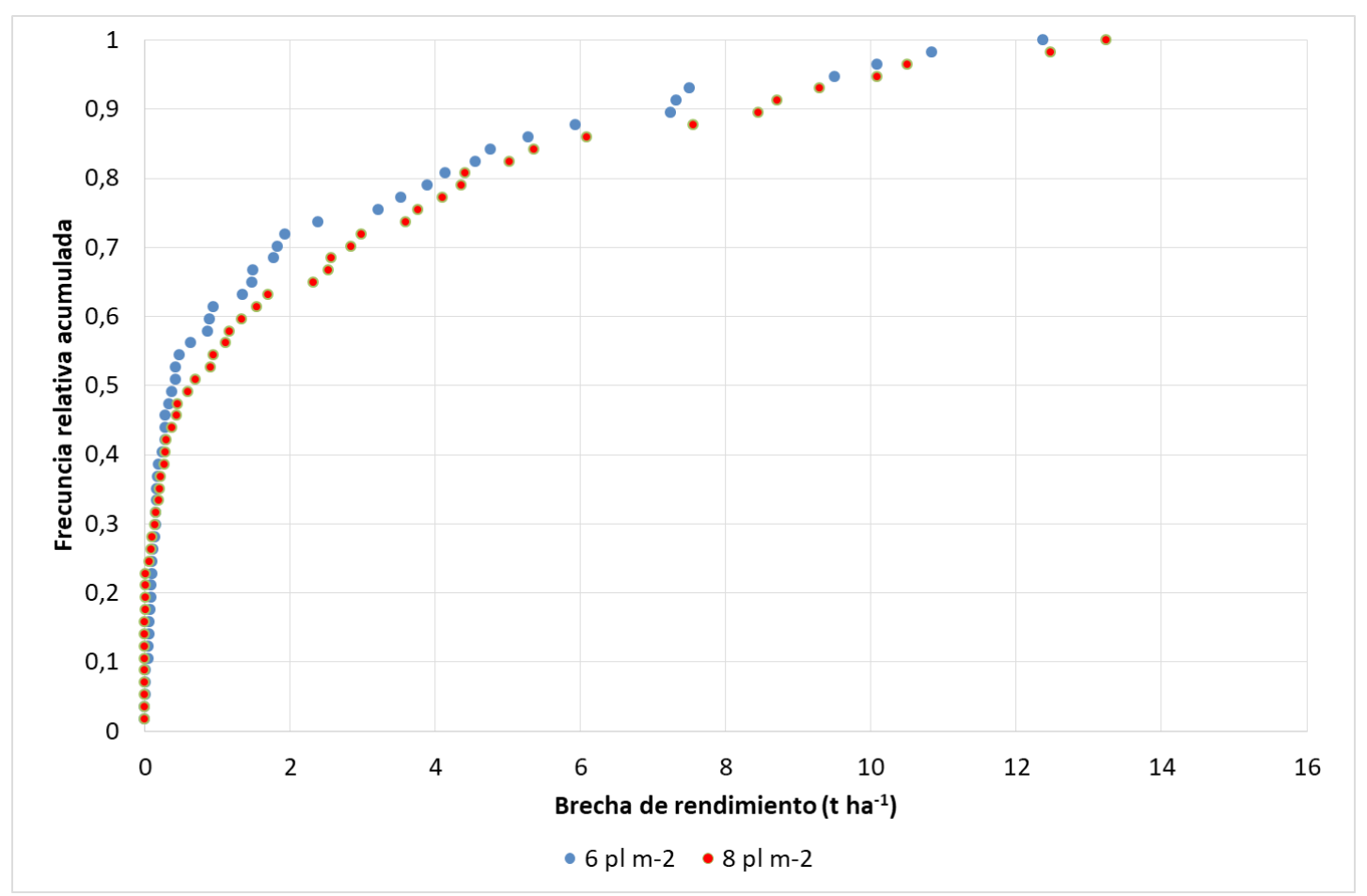

Figura 9. Frecuencia relativa acumulada de la brecha de rendimiento (BR) correspondiente al promedio de distintas fechas de siembra tardía del cultivo de maíz estimada entre 1960 y 2017 ( $n=57$ años) en Córdoba, Argentina, con distintas densidades de siembra (6 y 8 $\mathrm{pl} \mathrm{m} \mathrm{m}^{-2}$.

más conveniente que otra y recomendable eventualmente para reducir el riesgo de pérdidas más amplias en la región. En cambio, la diferencia que se suscita entre ambas densidades (6 y 8 pl $\mathrm{m}^{-2}$ ) en cada una de las oportunidades de siembra alcanza un carácter significativo sólo en tres de las seis comparaciones (sólo para las fechas de siembra fijas), que se expresa incrementando el valor medio de BR cuando la densidad es más elevada (Tabla 6). El valor de BR, en cambio, resulta indiferente de la DS cuando se analizan las fechas de siembra condicionadas a la acumulación de lluvia.

De esta forma, la siembra tardía de maíz en el centro de Córdoba para ambas poblaciones de plantas (6 y $8 \mathrm{pl} \mathrm{m}^{-2}$ ) no presenta BR diferentes en términos estadísticos relacionadas con un ligero anticipo o retraso y, por consiguiente, la condición de riesgo que promueve la diferencia de densidad puede ser comparada a partir de su comportamiento medio, como muestra la Figura 9. Mientras las BR nulas o muy pequeñas no presentan mayores diferencias, las curvas de probabilidad comienzan a diferenciarse recién a partir de valores de probabilidad empírica superiores a $40 \%$. La mayor densidad de siembra exhibe aproximadamente un $35 \%$ de probabilidad que BR supere el valor de $2 \mathrm{t} \mathrm{ha-1}$, en tanto este porcentaje se reduce casi a $28 \%$ en el caso de $6 \mathrm{pl} \mathrm{m}^{-2}$. Las BR más extremas (superiores a $8 \mathrm{t} \mathrm{ha}^{-1}$ ), en cambio, no muestran diferencias ostensibles asociadas con DS, de modo que la diferencia de población carece de interés cuando se presentan sequías extremas. La diferencia de probabilidad entre ambas densidades 
de siembra, que se acentúa para valores intermedios de BR (entre 0,5 y $8 \mathrm{t} \mathrm{ha}^{-1}$ ), pone de manifiesto que la condición de riesgo es ligeramente mayor cuando la densidad aumenta a $8 \mathrm{pl} \mathrm{m}^{-2}$. Además, la prueba de medias apareadas arroja una diferencia significativa ( $t=-2.75 ; \mathrm{P}<0,008)$ con un valor medio de BR que es mayor para el tratamiento de $8 \mathrm{pl} \mathrm{m}^{-2}$.

Incorporando algún elemento de costo al análisis, una referencia de interés es el costo de indiferencia que se interpreta como el rendimiento de maíz necesario para cubrir los costos de producción. Si bien el origen de esta información depende de los conceptos incluidos en el cálculo y la evolución del precio de insumos y productos con respecto al dólar, la consulta de una fuente local entre agosto de 2001 y agosto de 2017 para campo propio y en la zona núcleo asume un valor medio de 6,6 t ha-1 (Arbolave, 2018). A partir de este umbral, la probabilidad empírica de obtener rendimientos inferiores al costo de indiferencia es sólo de $12 \%$, y este resultado es indiferente respecto a la DS.

Sin embargo, esta visión tiene su contraparte cuando además de considerar el nivel de riesgo,

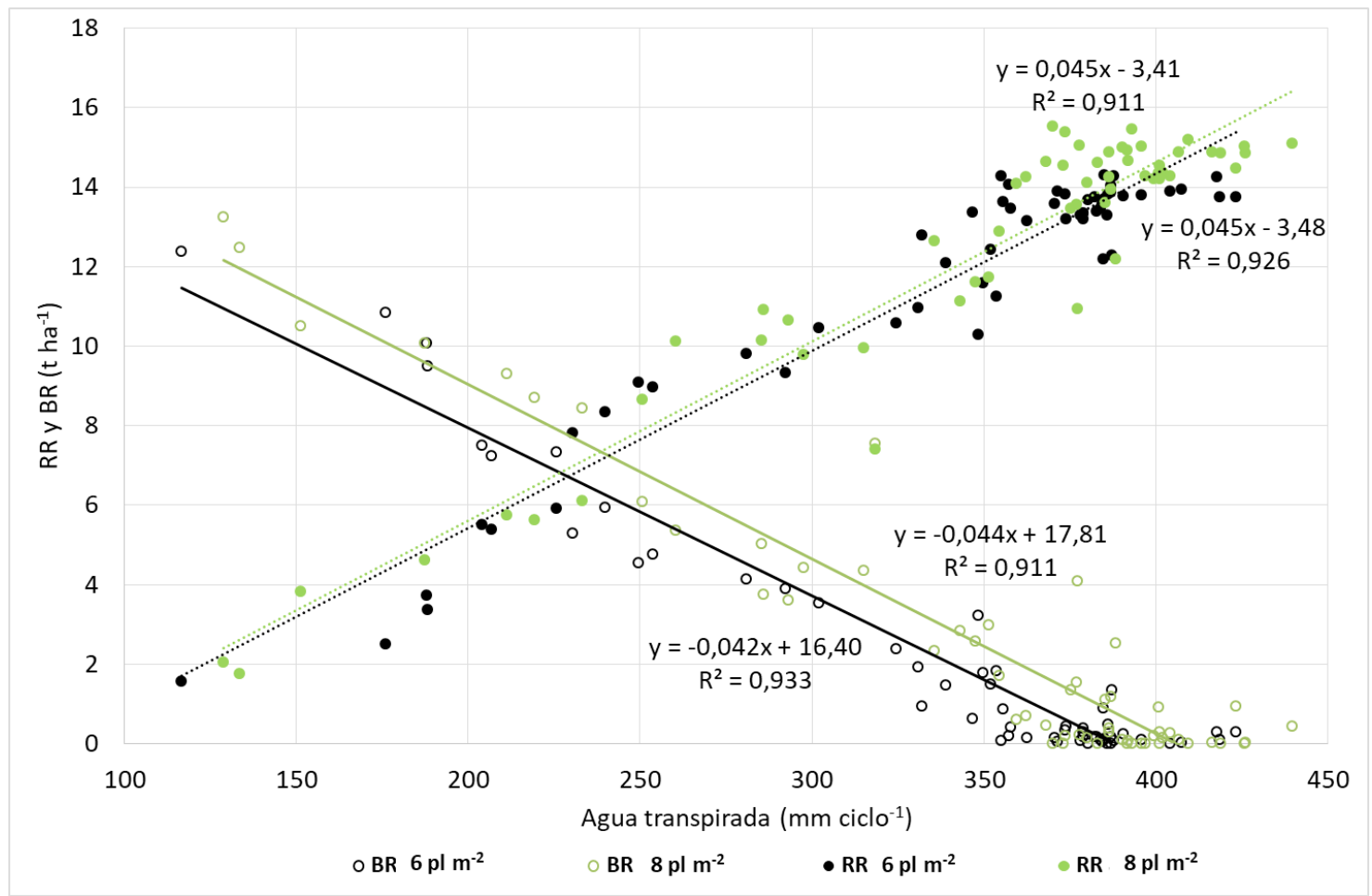

Figura 10. Relación del rendimiento efectivo (RR) y la brecha de rendimiento (BR) estimados con AquaCrop correspondientes al valor medio de las fechas de siembra del cultivo de maíz de siembra tardía bajo dos densidades de siembra, con respecto a la cantidad total de agua transpirada durante el ciclo.

se valora la productividad efectiva que estima AquaCrop. En este caso, la comparación de medias apareadas de los valores estimados de rendimiento efectivo para el conjunto de ciclos también alcanza una diferencia altamente significativa ( $t=-5,373 ; p<0,001)$ a favor de la mayor DS, con valores que superan en más del $82 \%$ de los años a los que se estiman con $6 \mathrm{pl} \mathrm{m}^{-2}$. En razón que el motor de crecimiento de AquaCrop está asociado directamente a la tasa de transpiración y que una curva de CC de mayor envergadura y más extendida en el tiempo representa un incremento del potencial productivo, los resultados de este análisis confirman que la disponibilidad de agua en la región no sería limitante para alcanzar este mayor potencial asociado al incremento de DS durante la mayoría de los años. La dependencia tanto de RR como de BR con respecto a la transpiración estimada por AquaCrop se presenta en la Figura 10.

De acuerdo al fundamento del modelo a los efectos de producir materia seca y rendimiento a partir del concepto de productividad del agua, el aumento de la cantidad total de agua transpirada 
por el cultivo de maíz durante su ciclo produce la disminución (aumento) aproximadamente lineal de $\mathrm{BR}(\mathrm{RR})$, de modo que la oferta de agua no resulta restrictiva para la producción de maíz tardío con una DS de $8 \mathrm{pl} \mathrm{m}{ }^{-2}$, siendo la brecha ligeramente mayor, pero alcanzando valores de RR más altos.

\section{CONCLUSIONES}

El método de Penman-Monteith, que calcula la tasa diaria de ETo empleando sólo registros diarios de temperatura máxima y mínima, sobreestima ligeramente los valores bajos y subestima los elevados; sin embargo explica alrededor del $80 \%$ (entre 76 y $86 \%$ ) de la variabilidad respecto del método original que utiliza el set completo de variables meteorológicas, con un error de estimación de aproximadamente $1 \mathrm{~mm}$ $\mathrm{d}^{-1}$. Utilizando registros térmicos observados a mayor distancia $(17 \mathrm{~km})$, el comportamiento de correlación y regresión resulta muy similar al que emplea los datos in situ, en tanto la pérdida de precisión del procedimiento simplificado sólo se incrementa marginalmente.

Calibrado para las condiciones de cobertura del cultivo, biomasa aérea y agua del suelo observadas en 2015-2016, el modelo AquaCrop constituye una herramienta apropiada para evaluar la brecha de rendimiento (BR) y el riesgo climático de producción de maíz tardío en la región central de Córdoba, Argentina. No se detectaron diferencias estadísticamente significativas para BR entre las distintas fechas de siembra tardía evaluadas (fijas y variables), tanto con $6 \mathrm{pl} \mathrm{m}^{-2}$ como para $8 \mathrm{pl} \mathrm{m}^{-2}$. En razón de no encontrar un comportamiento sistemático, ninguna oportunidad de siembra sería recomendable para reducir el nivel de riesgo en maíz bajo esta modalidad de siembra tardía. Asimismo, utilizando el valor medio de BR para caracterizar el nivel de peligro del cultivo de maíz tardío, se destaca en la región el predominio de un riesgo reducido, con un $70 \%$ de los ciclos productivos con valores inferiores a 2 t ha ${ }^{-1}$. Los años climáticamente desfavorables en particular por la ocurrencia de sequías agrícolas intensas, tienden a producir BR que son inviables económicamente en alrededor de $10 \%$ de los años.

El incremento de la densidad de siembra de 6 a $8 \mathrm{pl} \mathrm{m}^{-2}$ aumenta la magnitud de BR (riesgo) pero, asociado con la mayor tasa de transpiración, redunda en un manejo que es más productivo la mayoría de los años. La frecuencia de rendimientos inferiores a un costo de indiferencia estándar para la región alcanza un valor de $12 \%$, exactamente igual para ambas DS. La mayor DS, si bien conlleva un nivel de riesgo ligeramente mayor resulta también más productiva, lo que permite discernir que el agua disponible sería suficiente para sostener este manejo.

\section{BIBLIOGRAFIA}

Abedinpour, M., Sarangi, A., Rajput, T. B. S., Man Singh,Pathak, H. y Ahmad, T. (2012). Performance evaluation of AquaCrop model for maize crop in a semiarid environment. Agricultural Water Management, 110, 55-66.doi:10.1016/j.agwat.2012.04.001

Allen, R. G., Pereira, L. S., Raes, D. y Smith, M. (1998). Crop evapotranspiration: Guide-lines for computing crop water requirements. FAO Irrigation and Drainage Paper 56. Rome, Italy: Food and Agriculture Organization of the United Nations

Almorox, J, Senatore, A., Quej, V. H. y Mendicino, G. (2018). Worldwide assessment of the PenmanMonteith temperature approach for the estimation of monthly reference evapotranspiration. Theoretical and Applied Climatology,131 (1-2),693-703.doi: 10.1007/ s00704-016-1996-2

Andrade, F. H., Otegui, M. E. y Vega, C. (2000). Intercepted radiation at flowering and kernel number in maize. AgronomyJournal, 92, 92-97. doi:10.2134/ agronj2000.92192x

Arbolave, M. R. (2018). Proyecciones 2018/19 para soja, maíz y trigo. Márgenes Agropecuarios, 34(399), 1821.

Battisti, R., Sentelhas, P. C. y Boote, K. J. (2017). Inter-comparison of performance of soybean crop simulation models and their ensemble in southern Brazil. Field Crops Research, 200, 28-37.doi:10.1016/j. fcr.2016.10.004

Battisti, R., Sentelhas, P. C., Lopes Pascoalino, J. A., Sako, H., de SáDantas, J. P. y Ferreira Moraes, M. (2018). Soybean yield gap in the areas of yield contest in Brazil. International Journal of Plant Production, 12 (39), 159-168. doi: 10.1007/s42106-018-0016-0

Cantarero, M. G., Luque, S. F. y Rubiolo, O. J. (2000). Efecto de la época de siembra y la densidad de plantas sobre el número de granos y el rendimiento de un híbrido de maíz en la región central de Córdoba (Argentina). AGRISCIENTIA, 17, 3-10. doi:10.31047/1668.298x.v17.n0

Cerliani, C., Esposito, G. P., Morla, F. D., Balboa, G. R. y Naville, R. A. (2018). Relación entre la densidad óptima agronómica y el número de granos por planta en maíz (Zea mays L.). European Scientific Journal, 14(9), 1857-7881. doi:10.19044/esj.2018.v14n9p29

Cirilo, A. G. y Andrade, F. H. (1994). Sowing date and 
maize productivity: I. Crop growth and dry matter partitioning. Crop Science, 34 (4), 1039-1043. doi:10.2135/cropsci1994.0011183X003400040037x

Constantin, J., Willaume, M., Murgue, C., Lacroix, B. y Therond, O. (2015). The soil-crop models STICS and AqYield predict yield and soil watercontent for irrigated crops equally well with limited data. Agricultural and Forest Meteorology, 206, 55-68.doi:10.1016/j. agrformet.2015.02.011

Cox, W.J. (1996). Whole plant physiological and yield responses of maize to plant density. Agronomy Journal, 88 (3), 489-496.doi:10.2134/agronj1996.000 $21962008800030022 x$

Cross, H. Z. y Zuber, M. S.(1972). Prediction of flowering dates inmaize based on different methods of estimating thermal units.Agronomy Journal, 64 (3), 351-355.doi:10.2134/agronj1972.0002196200640003 $0029 x$

Dardanelli, J. D., Bachmeier, O. A., Sereno, R. y Gil, R. (1997). Rooting depth and soil water extraction patterns of different crops in a silty loam Haplustoll. Field CropsResearch, 54 (1), 29-38.doi:10.1016/ s0378-4290(97)00017-8

de la Casa, A., Ovando, G., Bressanini, L., Martínez, J., Díaz, G. y Miranda, C. (2018). Soybean crop coverage estimation from NDVI images with different spatial resolution to evaluate yield variability in a plot. ISPRS Journal of Photogrammetry and Remote Sensing,146,531-547.doi:10.1016/j. isprsjprs.2018.10.018

de la Casa, A., Ovando, G., Bressanini, L., Martínez, J., Miranda, C. y Díaz, G. (2016). Evaluación de un lote de maíz bajo riego y siembra variable con el modelo AquaCrop en Córdoba, Argentina.Revista Argentina de Agrometeorología, 7,1-13.

de la Casa, A. C. y Ovando, G. G. (2016). Variation of reference evapotranspiration in the central region of Argentina between 1941 and 2010. Journal of Hydrology: Regional Studies, 5, 66-79.doi: 10.1016/j. ejrh.2015.11.009

Djaman, K., Tabari, H., Balde, A. B., Diop, L., Futakuchi, K. e Irmak, S. (2016). Analyses, calibration and validation of evapotranspiration models to predict grass-reference evapotranspiration in the Senegal River delta. Journal of Hydrology: Regional Studies, 8, 82-94. doi: 10.1016/j.ejrh.2016.06.003

Donohue, R. J., McVicar, T. R. y Roderick, M. L. (2010). Assessing the ability of potential evaporation formulations to capture the dynamics in evaporative demand within a changing climate. Journalof Hydrology, $386 \quad$ (1-4), 186-197.doi:10.1016/j. jhydrol.2010.03.020

FAO (Food and Agriculture Organization of the United Nations). EToCalculator 2012 (versión 3.1). [Programa de cómputo]. Rome, Italy: Land and Water Division, FAO.

Flores-Gallardo, H., Ojeda-Bustamante, W., FloresMagdaleno, H., Sifuentes-lbarra, E. y Mejía-Saénz, E. (2013). Simulación del rendimiento de maíz (Zea mays L.) en el norte de Sinaloa usando el modelo AquaCrop. Agrociencia, 47 (4), 347-359.ISSN: 1405-3195.Recuperado de: https://www.redalyc.org/ articulo.oa?id=30226975004

Friedman, S. P. (2016). Evaluating the role of water availability in determining the yield-plant population density relationship. Soil ScienceSociety of America Journal, 80 (3), 563-578.doi:10.2136/ sssaj2015.11.0395

Gilmore, E. C. Jr. y Rogers, J. S. (1958). Heat units as a method ofmeasuring maturity in corn. Agronomy Journal, 50 (10), 611-615.doi:10.2134/agronj1958.000 $21962005000100014 x$

Grassini, P., Yang, H. y Cassman, K. G. (2009). Limits to maize productivity in Western Corn-Belt: A simulation analysis for fully irrigated and rainfed conditions. Agricultural and ForestMeteorology, 149 (8), 12541265.doi:10.1016/j.agrformet.2009.02.012

Grassini, P., Thorburn, J., Burr, C. y Cassman, K. G. (2011). High-yield irrigated maize in the Western U.S. Corn Belt: I. On-farm yield, yield potential, and impact of agronomic practices. Field Crops Research, 120 (1), 142-150.doi:10.1016/j.fcr.2010.09.012

Heng, L. K., Hsiao, T. C. Evett, S.R. Howell, T.A. y Steduto, P. (2009). Validating the FAO AquaCrop Model for Irrigated and Water Deficient Field Maize. Agronomy Journal. Agronomy Journal, 101 (3), 488-498. doi:10.2134/agronj2008.0029xs

Hsiao, T. C., Heng, L. K., Steduto, P., Rojas-Lara, B., Raes, D. y Fereres, E. (2009). AquaCrop-The FAO crop model to simulate yield response to water: III. Parameterization and testing for maize. Agronomy Journal, 101, 448-459.doi:10.2134/agronj2008.0218s

Li, J., Xie, R. Z., Wang, K. R., Ming, B., Guo, Y. Q., Zhang, G. Q. y Li, S. K. (2015). Variations in maize dry matter, harvest index, and grain yield with plant density. Agronomy Journal, 107 (3),829-834.doi:10.2134/ agronj14.0522

Lobell, D. B., Cassman, K. G. y Field, C. B. (2009). Crop yield gaps: their importance, magnitude, and causes. Annual Review of Environment and Resources, 34, 179204. doi: 10.1146/annurev.environ.041008.093740

Irmak, S., Kabenge, I., Skaggs, K. E. y Mutiibwa, D. (2012). Trend and magnitude of changes in climate variables and reference evapotranspiration over 116yr period in the Platte River Basin, Central Nebraska, USA. Journal of Hydrology, 420 (4), 228-244.doi: 10.1016/j.jhydrol.2011.12.006

McVicar, T. R., Roderick, M. L., Donohue, R. J., Li, L. 
T., Van Niel, T. G., Thomas, A., Grieser, J., Jhajharia, D., Himri, Y., Mahowald, N. M., Mescherskaya, A. V., Kruger, A. C., Rehman, S. y Dinpashoh, Y. (2012). Global review and synthesis of trends in observed terrestrial near-surface wind speeds: Implications for evaporation. Journal ofHydrology, 416-417, 182-205. doi: 10.1016/j.jhydrol.2011.10.024

McMaster, G. S. y Wilhelm, W. W. (1997). Growing degreedays: one equation, two interpretations. Agricultural and Forest Meteorology, 87, 291-300.doi: 10.1016/ S0168-1923(97)00027-0

Méndez, M. J., Casagrande, G. y Vergara, G. (2014). Balance hídrico del cultivo de maíz en siembras de primavera temprana y tardía en la región semiárida argentina. Documento presentado en laReunión Binacional Uruguay-Argentina de Agrometeorología. Piriápolis, Uruguay.

Raes, D., Steduto, P., Hsiao, T. C. y Fereres, E. (2009). AquaCrop-The FAO crop model to predict yield response to water: II Main algorithms and software description. Agronomy Journal, 101, 438-447. doi:10.2134/agronj2008.0140s

Sangoi, L., Gracietti, M. A., Rampazzo, C. y Bianchetti, P. (2002). Response of Brazilian maize hybrids from different eras to changes in plant density. Field Crops Research, 79 (1), 39-51. doi:10.1016/S03784290(02)00124-7

Sarlangue, T., Andrade, F. H., Calviño, P. A. y Purcell. L.C. (2007). Why do maize hybrids respond differently to variations in plant density? Agronomy Journal, 99, 984-991. doi:10.2134/agronj2006.0205

Steduto, P., Hsiao, T. C., Raes, D. y Fereres, E. (2009). AquaCrop- the FAO crop model to simulate yield response towater: I. Concepts and underlying principles. Agronomy Journal, 101 (3), 426-437. doi:10.2134/agronj2008.0139s

Stricevic, R., Cosic, M., Djurovic, N., Pejic, B. y Maksimovic, L. (2011). Assessment of the FAO AquaCrop model in the simulation of rainfed and supplementally irrigated maize, sugar beet and sunflower. Agricultura/Water Management, 98 (10), 1615-1621.doi:10.1016/j. agwat.2011.05.011

Vicente-Serrano, S. M., Bidegain, M., Tomas-Burguera, M., Dominguez-Castro, F., El Kenawy, A., McVicar, T. R., Azorin-Molina, C., López-Moreno, J. I., Nieto, R., Gimeno, L. y Giménez, A. (2017). A comparison of temporal variability of observed and model-based pan evaporation over Uruguay (1973-2014). International Journal of Climatology,38(1), 337-350. doi:10.1002/ joc.5179

Willey, R. W. y Heath, S. B. (1969). The quantitative relationships between plant population and crop yield. Advances in Agronomy, 21, 281-321. doi:10.1016/ S0065-2113(08)60100-5

Willmott, C. J., Robeson, S. M. y Matsuura, K. (2012). A refined index of model performance. International Journal of Climatology, 32 (13), 2088-2094.doi: 10.1002/joc.2419 